\section{Grau de atividade física e síndrome metabólica: um estudo transversal com indígenas Khisêdjê do Parque Indígena do Xingu, Brasil}

\author{
Degree of physical activity and metabolic syndrome: \\ a cross-sectional study among the Khisêdjê group \\ in the Xingu Indigenous Park, Brazil
}

\author{
1 Departamento de Medicina \\ Preventiva, Universidade \\ Federal de São Paulo, São \\ Paulo, Brasil. \\ Correspondência \\ K. M. Santos \\ Departamento de Medicina \\ Preventiva, Universidade \\ Federal de São Paulo. \\ Rua Borges Lagoa 1341 \\ 2o andar, São Paulo, SP \\ 04038-034, Brasil. \\ kmasekel@gmail.com
}

\begin{abstract}
This study aimed to verify the existence of an association between degree of physical activity and presence of metabolic syndrome in the Khisêdjê indigenous group. The authors evaluated $170 \mathrm{in}$ dividuals 20 years or older, based on demographic data, physical examination, and laboratory tests. The data were analyzed with the chi-square test ( $p<0.05)$, crude and adjusted prevalence ratios (point and 95\% confidence intervals), and Student's t-test. Satisfactory results were observed in relation to cardiorespiratory endurance, flexibility, bending of arms and trunk, and measurement of physical activity according to the number of steps/day. Prevalence of metabolic syndrome was 27.8\% and was higher in women, the 39-49-year and $\geq 50$-year age groups, and in individuals with lower performance on the cardiorespiratory endurance test, horizontal impulse, and number of steps/day. The results indicate the need for greater surveillance in the control and prevention of risk factors for metabolic syndrome.
\end{abstract}

Metabolic Syndrome X; Motor Activity; Indigenous Population
Kennedy Maia dos Santos 1

Mario Luiz da Silva Tsutsui 1

Patrícia Paiva de Oliveira Galvão 1

Lalucha Mazzucchetti 1

Douglas Rodrigues 1

Suely Godoy Agostinho Gimeno ${ }^{1}$

\section{Introdução}

A síndrome metabólica é considerada um conjunto de fatores de risco que está diretamente relacionado ao aumento do risco de doenças cardiovasculares e diabetes 1. Estima-se que $20 \%$ a $25 \%$ da população adulta do mundo tenha essa síndrome 1 . No Brasil, até o presente, não existem dados precisos sobre a prevalência geral da síndrome metabólica, havendo grandes diferenças de acordo com a população e local de estudo. Pesquisas epidemiológicas identificaram prevalências que variam de $8,6 \%$, na população em geral, e até $89 \%$, em grupo de pessoas com diabetes 2,3 .

Nos últimos anos, observa-se, na literatura, crescente interesse no aumento da prevalência da síndrome metabólica também entre as populações indígenas. As prevalências entre indígenas no mundo variam de $10 \%$, em indígenas da Sibéria, a 33\%, em australianos 4,5. No Brasil, alguns estudos mostram situação ainda mais preocupante, com valores de até $65,3 \%$ entre os Kaingáng 6 .

Estudos indicam que o processo de ocidentalização contribui para a ocorrência desses agravos entre os povos indígenas 7,8,9. Sabe-se, por exemplo, que a maior proximidade destes com as populações urbanas associa-se às modificações de sua relação com a terra, o trabalho e a alimentação 10. Essas mudanças trazem consigo uma consequente alteração no perfil epidemio- 
lógico desses povos, sendo possível observar elevação de agravos à saúde, tais como, obesidade, hipertensão, intolerância à glicose, diabetes e síndrome metabólica 8,9,11. Sabe-se que fatores comportamentais, entre eles, hábitos alimentares inadequados e inatividade física, que estão associados à maior prevalência desses agravos nas populações urbanas, estão se tornando comuns também entre diferentes povos indígenas 8,9,12,13.

Modificações no estilo de vida, com a adoção de hábitos saudáveis, podem exercer um impacto considerável na redução da prevalência das doenças crônicas não transmissíveis (DCNT) gerais e da síndrome metabólica; nesse sentido, a atividade física desempenha importante papel na manutenção do bem-estar, na prevenção e no controle das DCNT 14,15. Vuori 16 destaca que essa prática favorece o metabolismo lipídico, auxilia no controle da pressão arterial e da densidade óssea, além de contribuir positivamente nas funções psicológicas e imunológicas. Outros efeitos constatados incluem a redução do peso corporal; melhoria da função cardiorrespiratória, favorecendo a manutenção dos níveis normais de frequência cardíaca; elevação da captação de glicose pelo músculo ativo durante e após a sessão de exercício, melhorando a sensibilidade da ação da insulina 17,18,19. Katzmarzyk et al. 14 observaram redução de $30 \%$ na prevalência da síndrome metabólica em população canadense saudável após intervenção com a introdução de exercícios predominantemente aeróbios.

Embora a associação entre atividade física e ocorrência de síndrome metabólica já tenha sido identificada por meio de estudos epidemiológicos, essa relação ainda não foi estudada entre as populações indígenas do Brasil. Dentro desse contexto, a proposta do presente trabalho é verificar a existência de associação entre desempenho físico e a presença de síndrome metabólica entre índios Khisêdjê, do Parque Indígena do Xingu.

\section{Material e métodos}

Trata-se de estudo epidemiológico transversal, que incluiu indivíduos residentes na região medial do Parque Indígena do Xingu pertencentes à etnia Khisêdjê, de ambos os sexos, com idade $\geq 20$ anos. A existência de infraestrutura de apoio e de profissionais da saúde (enfermeira, médico e dentista) que prestam atendimento permanente a esses sujeitos, bem como a possibilidade de acesso por via terrestre foram alguns dos fatores que contribuíram para a escolha da comunidade Khisêdjê para a realização desta pesquisa.

O povo Khisêdjê (outrora chamados de Suyá) é falante da língua Jê. Atualmente, vivem em quatro aldeias Khisêdjê e totalizam cerca de 400 sujeitos (Fundação Nacional de Saúde. REDEFUNASA: quantitativo de pessoas. http:// sis.funasa.gov.br/transparencia_publica/sia siweb/Layout/quantitativo_de_pessoas_2010. asp, acessado em Nov/2010).

Dos 190 indivíduos com 20 anos ou mais (47\% da população), 181 sujeitos participaram desta investigação (95,3\%). Entre os participantes, 11 mulheres foram excluídas por não haver informações relativas às avaliações físicas (quatro grávidas e sete por estarem com alguma debilidade na saúde).

A coleta de dados foi realizada na aldeia principal (Ngojwere), no posto indígena Wawi, e ocorreu em dois momentos: em 2010 (julho) e 2011 (agosto/setembro). Profissionais treinados (médicos, enfermeiras, nutricionistas e educadores físicos) coletaram as informações de interesse (dados sociodemográficos, realização de exames e testes).

A pressão arterial (sistólica - PAS e diastólica - PAD) foi aferida com aparelho automático de pulso (modelo HEM 742 INT; OMRON Healthcare, China), em triplicata, após repouso por dez minutos, em posição sentada. O valor final foi a média aritmética das duas últimas medidas. Utilizou-se como critério diagnóstico o proposto no relatório do Joint National Commitee on Prevention, Detection, Evaluation, and Treatment of High Blood Pressure 20 para adultos maiores de 18 anos, a saber: normal - PAS < 120mmHg e $\mathrm{PAD}<80 \mathrm{mmHg}$; pré-hipertenso - PAS entre 120 $139 \mathrm{mmHg}$ e PAD entre $80-89 \mathrm{mmHg}$; hipertenso - valores de PAS ou PAD, respectivamente, $\geq$ $140 \mathrm{mmHg}$ ou $\geq 90 \mathrm{mmHg}$.

O perímetro da cintura foi verificado em duplicata, utilizando-se fita métrica metálica (modelo 17; Grafco, São Paulo, Brasil) para obtenção do valor da menor curvatura localizada entre as costelas e a crista ilíaca. O valor final foi a média aritmética entre as medidas. A presença de obesidade central foi caracterizada por valores de perímetro de cintura $\geq 94 \mathrm{~cm}$, para homens, ou $\geq 80 \mathrm{~cm}$, para mulheres 21 .

O teste oral de tolerância à glicose (TOTG) foi feito, após jejum de 12 horas, com glicemia capilar de jejum (GJ) e de duas horas (G2h), após a ingestão de solução com $75 \mathrm{~g}$ de glicose (Glutol sabor guaraná garrafa pet $300 \mathrm{~mL}$, sem gás; Laborclin Produtos para Laboratórios, Pinhais, Brasil). OTOTG foi precedido de rastreamento para o diabetes mellitus; seis indivíduos com diagnóstico da doença foram dispensados de realizar o teste. Para classificação dos diferentes graus de tolerância à glicose, utilizaram-se os pontos de corte propostos pela American Diabetes Association 22 , com as correções para aferições em 
sangue capilar indicadas pela Organização Mundial da Saúde 23. Os indivíduos foram considerados normais quando não tinham história prévia de diabetes mellitus e cujas dosagens de GJ e G2h apresentassem, respectivamente, valores $<100 \mathrm{mg} / \mathrm{dL}$ ou $<140 \mathrm{mg} / \mathrm{dL}$. Aqueles com história prévia de diabetes mellitus ou com glicemia de jejum $\geq 200 \mathrm{mg} / \mathrm{dL}$ foram classificados como diabéticos. Os participantes foram classificados com glicemia de jejum alterada (GJA) desde que não referissem história prévia de diabetes mellitus e apresentassem $\mathrm{GJ} \geq 100 \mathrm{mg} / \mathrm{dL}$ e $<110 \mathrm{mg} / \mathrm{dL}$ e G2h < 140mg/dL. Na classe tolerância à glicose diminuída (TGD), foram incluídos os que, sem história prévia de diabetes mellitus, apresentaram GJ < 110mg/dL e valores de G2h $\geq 140 \mathrm{mg} / \mathrm{dL}$ e $<200 \mathrm{mg} / \mathrm{dL} 23$.

Uma amostra de sangue venoso colhida após jejum de 12 horas foi obtida para dosar a concentração de lipoproteínas séricas. Os níveis de HDL e triglicérides foram determinados por kits enzimáticos e processados em analisador automático. Os valores de referência para identificação da hipertrigliceridemia e HDL reduzido foram, respectivamente, triglicérides > 150mg/dL e HDL $<40 \mathrm{mg} / \mathrm{dL}$, para homens, ou $<50 \mathrm{mg} / \mathrm{dL}$, para mulheres 21 .

Para o diagnóstico de síndrome metabólica, utilizou-se o critério resultante do consenso estabelecido pela International Diabetes Federation Task Force on Epidemiology and Prevention; National Heart, Lung, and Blood Institute; American Heart Association; World Heart Federation; International Atherosclerosis Society e pela International Association for the Study of Obesity. O consenso propõe a presença de pelo menos três entre os cinco fatores de risco: hipertensão arterial, intolerância à glicose ou diabetes mellitus, presença de obesidade central, hipertrigliceridemia ou HDL reduzido 21 .

Foram aplicados testes para identificação do condicionamento físico e nível de atividade física. No teste de Rockport (ou de uma milha) os sujeitos foram orientados a caminhar, o mais rápido possível, por uma distância de 1.609 metros. Ao final da prova, registrou-se a sua duração (minutos; cronômetro da marca Guepardo modelo OF0200; Guepardo, Camboriú, Brasil) e a frequência cardíaca do indivíduo (frequencímetro da marca e modelo Oregon Scientific HR-102; IDT Tecnology Limited, China). O volume máximo de oxigênio $\left(\mathrm{VO}_{2 \max }\right)$, indicativo da aptidão cardiorrespiratória, foi obtido pela equação do Rockport Walking Institute 17. Os critérios utilizados para classificação do $\mathrm{VO}_{2 \max }$ foram os propostos pela American Heart Association 24.

Para avaliação da flexibilidade, utilizou-se o Banco de Wells (sentar e alcançar - TSA/Teste de Wells \& Dillon). Durante o procedimento, o indivíduo sentava-se, em uma superfície plana, de frente para o banco, com os joelhos estendidos, apoiando os pés no banco em flexão plantar. Nesse momento, os pés localizavam-se abaixo do prolongamento do banco, no 23o centímetro da escala métrica 25. Com membros superiores estendidos e tronco flexionado, registrou-se o maior valor de três tentativas, tendo como referência a ponta dos dedos. Para classificação dos resultados nesse teste, empregou-se o critério proposto por Pollock \& Wilmore 26 .

O teste de impulso horizontal foi aplicado para avaliar a força muscular de membros inferiores. Foi utilizada uma trena aberta de fibra de vidro de 50m (Profield de La Cruz do Brasil, São Paulo, Brasil) para medir a distância do salto, além de uma fita afixada ao chão, demarcando o local onde o indivíduo se posicionava para a execução da tarefa. Com os pés ligeiramente afastados, pontas dos pés sobre a fita sem ultrapassá-la, joelhos e tronco semiflexionados, os sujeitos executavam três saltos, sendo considerado apenas o melhor deles. A classificação dos resultados seguiu o critério recomendado por Caldas \& Rocha 27.

O teste de flexão de tronco foi utilizado para avaliar a resistência muscular localizada de membros superiores. Empregou-se o procedimento sugerido pela American College of Sports Medicine 28. Fitas adesivas foram fixadas no solo transversalmente ao lado de um colchonete; deuse distância de $8 \mathrm{~cm}$ da primeira para a segunda fita, para o teste dos sujeitos com idade $\geq 45$ anos, e de $12 \mathrm{~cm}$ da primeira para a terceira fita, para o daqueles com idade $<45$ anos. O indivíduo deitava-se em decúbito dorsal, com joelhos ligeiramente flexionados a $90^{\circ}$, braços e dedos estendidos, dedos posicionados em cima da primeira fita fixada. $\mathrm{O}$ avaliado executava as abdominais compassadamente, havendo validação quando a ponta dos dedos percorria a distância entre as fitas correspondente à faixa etária. O teste encerrava-se quando o indivíduo executava seu maior número de repetições ou quando completava 75 execuções. A classificação nesse teste seguiu a proposta da Canadian Society for Phisiology and Fitness Canada 28.

O teste de flexão de braço foi empregado para avaliar a resistência muscular localizada de membros superiores. Foram adotados o critério e o protocolo do Canadian Standardized Test of Fitness (CSTF) 29. As mulheres apoiavam os joelhos e mãos no solo; os homens apoiavam as pontas dos pés. Com braços ligeiramente à frente, distantes um do outro, acompanhando a amplitude dos ombros e com cotovelos estendidos, executavam o maior número de repetições até a exaustão. Uma execução completa correspondia 
a um movimento em que os cotovelos eram flexionados (cotovelo a $90^{\circ}$ aproximadamente) e se estendiam completamente, conforme descrição da CSTF. A classificação no teste ocorreu mediante o número de repetições executadas, segundo sexo e faixa etária 29.

O nível de atividade física conforme o número de passos/dia foi medido em três dias consecutivos, por meio do pedômetro (modelo DISTA F100; Geonaute, China). Após medição do tamanho médio do passo do indivíduo, colocava-se o equipamento ao lado direito da cintura, devendo ser retirado apenas para dormir e para o banho. A cada 24 horas, o número de passos era registrado. A classificação (média de três dias) foi feita de acordo com a proposta de Tudor-Locke et al. 30: < 5.000 passos/dia - indivíduos sedentários; de 5.000 a 7.499 passos/dia - com baixa atividade; de 7.500 a 9.999 passos/dia - pouco ativos (mínimo satisfatório); de 10.000 a 12.499 passos/dia - plenamente ativos; 12.500 passos/dia ou mais -muito ativos.

Com o objetivo de realizar uma avaliação mais abrangente do desempenho dos indígenas nos testes físicos, criou-se uma variável denominada "desempenho físico global". A variável foi composta com base nos resultados nos testes de Rockport, flexibilidade, flexão de tronco, flexão de braço. Foram considerados com desempenho físico global satisfatório aqueles indivíduos que obtiveram desempenho bom, acima da média ou excelente em, no mínimo, três testes; os demais foram classificados como tendo desempenho físico global insatisfatório.

A existência de associação entre atividade física e a presença de síndrome metabólica foi avaliada pela estatística qui-quadrado $(\mathrm{p}<0,05)$ e pelas razões de prevalências (por ponto e por intervalo de 95\% de confiança). Para obtenção da razão de prevalência ajustada (por sexo e idade), utilizou-se a regressão de Poisson. Empregou-se, para a comparação dos valores médios das variáveis biológicas dos indivíduos, segundo sexo ou idade, o teste t de Student 31. O programa Stata (Stata Corp. College Station, Estados Unidos) foi utilizado em todas as etapas da análise.

Este estudo foi aprovado pelo Comitê de Ética em Pesquisa da Universidade Federal de São Paulo (UNIFESP - parecer de número 0320/2011) e pela Comissão Nacional de Ética em Pesquisa (CONEP - parecer de número 113/2011).

\section{Resultados}

Entre os 170 indivíduos que participaram do estudo, $40 \%$ eram do sexo feminino $(n=68)$. A média de idade foi de 38 anos (desvio-padrão -
DP $=14,8$ anos). Em ambos os sexos, observou-se maior porcentagem de indígenas na faixa etária de 20 a 29 anos $(38,2 \%)$.

Em relação à atividade física (Tabela 1), diferenças estatisticamente significantes foram encontradas entre os valores médios de todos os testes, segundo sexo $(\mathrm{p}<0,05)$. As mulheres apresentaram médias superiores nos testes de flexibilidade e flexão de braço, enquanto os homens obtiveram médias maiores nos testes de flexão de tronco, impulso horizontal, número de passos/ dia e no teste de uma milha $(\mathrm{p}<0,05)$.

Quanto ao desempenho físico, observou-se que $90,7 \%$ e $73 \%$ dos indígenas apresentaram desempenho bom ou excelente no teste de uma milha e no teste de flexibilidade, respectivamente. Nos testes de flexão de braço e tronco, 50,5\% e $33,3 \%$, respectivamente, foram classificados com desempenho acima da média ou excelente. Entretanto, o mesmo não ocorreu no teste de impulso horizontal, no qual apenas $1,1 \%$ apresentou desempenho bom. Entre aqueles que participaram da avaliação com o pedômetro, $48,4 \%$ foram classificados como muito ativos (Tabela 1).

A prevalência de síndrome metabólica foi $27,8 \%$, sendo esta maior entre as mulheres quando comparadas aos homens (Tabela 2; $\mathrm{p}=0,003$ ). Quanto aos componentes da síndrome metabólica, as prevalências foram: hipertensão arterial, 6,8\%; intolerância à glicose, 23,1\%; obesidade central, $37,4 \%$; hipertrigliceridemia, 43,5\%; HDL-c reduzido, 66,2\%. As médias dos componentes da síndrome metabólica podem ser observadas na Tabela 2.

Ao comparar os valores médios das variáveis relativas à atividade física segundo a presença de síndrome metabólica (Tabela 3), verificou-se que os indivíduos sem essa condição apresentaram melhores resultados no teste de uma milha $(\mathrm{p}<0,001)$, número de passos/dia $(\mathrm{p}=0,004)$ e impulso horizontal $(p=0,018)$. O número e a porcentagem de indígenas segundo a presença de síndrome metabólica e prática de atividade física podem ser observados na Tabela 3. Pode-se verificar que a porcentagem de síndrome metabólica foi superior entre os sujeitos com idade $\geq$ 30 anos ( $\mathrm{p}<0,001)$, em comparação à observada entre os mais jovens; o mesmo se deu no sexo feminino em relação ao masculino. Além disso, a prevalência de síndrome metabólica também foi estatisticamente maior nos sujeitos que tiveram desempenho fraco ou regular no teste de uma milha (Rockport), quando comparados com aqueles que apresentaram desempenho excelente. Situação semelhante foi observada no que se refere à avaliação do nível de atividade física por meio do número de passos/dia (com pedômetro) e entre os sujeitos classificados como sedentá- 
Número (ou média) e porcentagem (ou desvio-padrão - DP) de indígenas Khisêdjê segundo variáveis relativas à atividade física, por sexo. Parque Indígena do Xingu, Brasil, 2011.

\begin{tabular}{|c|c|c|c|c|c|c|c|}
\hline \multirow[t]{3}{*}{ Variável } & \multicolumn{4}{|c|}{ Sexo } & \multicolumn{2}{|c|}{ Total $(n=170)$} & \multirow[t]{3}{*}{ Valor de $p$ * } \\
\hline & \multicolumn{2}{|c|}{ Feminino $(n=68)$} & \multicolumn{2}{|c|}{ Masculino $(n=102)$} & \multirow[b]{2}{*}{ n (ou média) } & \multirow[b]{2}{*}{$\%$ (ou DP) } & \\
\hline & n (ou média) & \% (ou DP) & n (ou média) & $\%$ (ou DP) & & & \\
\hline Idade (anos) & 40,0 & 15,9 & 36,6 & 13,9 & 38,0 & 14,8 & 0,066 \\
\hline Teste de uma milha $\left(\mathrm{VO}_{2} \max ; \mathrm{mL} / \mathrm{kg} / \mathrm{min}\right)$ & $46(38,0)$ & 7,87 & $94(45,8)$ & 6,8 & $140(43,3)$ & 8,0 & $<0,001$ \\
\hline Fraco/Regular ** & 6 & 13,0 & 7 & 7,4 & 13 & 9,3 & 0,016 \\
\hline Bom & 32 & 69,5 & 48 & 51,0 & 80 & 57,1 & \\
\hline Excelente & 8 & 17,3 & 39 & 41,4 & 47 & 33,5 & \\
\hline Teste de flexibilidade [banco de & $55(38,5)$ & 5,19 & $97(35,1)$ & 6,6 & $152(36,3)$ & 6,3 & $<0,001$ \\
\hline \multicolumn{8}{|l|}{ Wells] (cm) } \\
\hline Regular/Médio *** & 12 & 21,8 & 29 & 29,9 & 41 & 27,0 & 0,020 \\
\hline Bom & 11 & 20,0 & 34 & 35,0 & 45 & 29,6 & \\
\hline Excelente & 32 & 58,2 & 34 & 35,0 & 66 & 43,4 & \\
\hline Teste de flexão de braço (repetições) & $19(32,4)$ & 20,0 & $68(22,9)$ & 8,2 & $87(25,0)$ & 12,3 & 0,001 \\
\hline Fraco \# & 3 & 15,8 & 4 & 5,9 & 7 & 8,0 & 0,002 \\
\hline Abaixo da média & 0 & 0 & 14 & 20,5 & 14 & 16,1 & \\
\hline Médio & 2 & 10,5 & 20 & 29,4 & 22 & 25,3 & \\
\hline Acima da média & 3 & 15,8 & 17 & 25,0 & 20 & 23,0 & \\
\hline Excelente & 11 & 57,9 & 13 & 19,1 & 24 & 27,5 & \\
\hline Teste de flexão de tronco (repetições) & $18(20,4)$ & 22,4 & $70(31,9)$ & 17,4 & $88(29,6)$ & 19,0 & 0,010 \\
\hline Fraco \#\# & 7 & 41,1 & 6 & 8,5 & 13 & 15,0 & 0,017 \\
\hline Abaixo da média & 2 & 11,7 & 13 & 18,5 & 15 & 17,2 & \\
\hline Médio & 4 & 23,5 & 26 & 37,1 & 30 & 34,5 & \\
\hline Acima da média & 2 & 11,7 & 18 & 25,7 & 20 & 23,0 & \\
\hline Excelente & 2 & 11,7 & 7 & 10,0 & 9 & 10,3 & \\
\hline Teste de impulso horizontal (metros) & $24(1,1)$ & 0,2 & $69(1,8)$ & 0,3 & $93(1,6)$ & 0,4 & $<0,001$ \\
\hline Fraco \#\#\# & 24 & 100 & 65 & 94,2 & 89 & 95,7 & 0,483 \\
\hline Regular & 0 & 0 & 3 & 4,3 & 3 & 3,2 & \\
\hline Bom & 0 & 0 & 1 & 1,4 & 1 & 1,0 & \\
\hline Número de passos/dia & $18(10.645,9)$ & $2.915,7$ & $46(12.977,8)$ & $4.409,0$ & $64(12.321,9)$ & $4.159,0$ & 0,021 \\
\hline Sedentário/Com baixa atividade $\S$ & 1 & 5,5 & 2 & 4,3 & 3 & 4,7 & 0,055 \\
\hline Pouco ativo & 6 & 33,3 & 10 & 21,7 & 16 & 25,0 & \\
\hline Ativo & 7 & 38,9 & 7 & 15,22 & 14 & 21,9 & \\
\hline Muito ativo & 4 & 22,2 & 27 & 58,7 & 31 & 48,4 & \\
\hline
\end{tabular}

$\mathrm{VO}_{2 \mathrm{max}}$ : volume máximo de oxigênio.

* Referente ao teste qui-quadrado ou t de Student;

** De acordo com Pitanga 24;

*** De acordo com Pollock \& Wilmore 26;

\# De acordo com Nieman (1995, apud Morrow et al. 29);

\#\# De acordo com American College of Sports Medicine 28;

\#\#\# De acordo com Caldas \& Rocha 27;

$\S$ De acordo com Tudor-Locke et al. 30.

rios/com baixa atividade e pouco ativos, quando comparados com aqueles do grupo classificado como muito ativo.

Em relação à variável desempenho físico global, observou-se que $53 \%$ dos indivíduos obti- veram desempenho satisfatório (desempenho bom ou superior em pelo menos três dos quatros testes físicos). Ademais, não se observou associação desta variável com a presença de síndrome metabólica (Tabela 3). 
Tabela 2

Número (ou média) e porcentagem (ou desvio-padrão - DP) de indígenas Khisêdjê segundo presença de síndrome metabólica e seus componentes, por sexo. Parque Indígena do Xingu, Brasil, 2011.

\begin{tabular}{|c|c|c|c|c|c|c|c|}
\hline \multirow[t]{3}{*}{ Variável } & \multicolumn{4}{|c|}{ Sexo } & \multicolumn{2}{|c|}{ Total $(n=170)$} & \multirow[t]{3}{*}{ Valor de $p$ * } \\
\hline & \multicolumn{2}{|c|}{ Feminino $(n=68)$} & \multicolumn{2}{|c|}{ Masculino $(n=102)$} & \multirow[b]{2}{*}{ n (ou média) } & \multirow[b]{2}{*}{ \% (ou DP) } & \\
\hline & n (ou média) & \% (ou DP) & n (ou média) & \% (ou DP) & & & \\
\hline PAS (mmHg) & 114,6 & 17,3 & 122,7 & 13,4 & 119,5 & 15,5 & $<0,001$ \\
\hline PAD $(\mathrm{mmHg})$ & 65,6 & 9,1 & 64,7 & 8,3 & 65,1 & 8,6 & 0,240 \\
\hline \multicolumn{8}{|l|}{ Hipertensão arterial ** } \\
\hline $\operatorname{Sim}$ & 5 & 7,8 & 6 & 6,1 & 11 & 6,8 & 0,676 \\
\hline Não & 59 & 92,2 & 92 & 93,9 & 151 & 93,2 & \\
\hline $\mathrm{GJ}(\mathrm{mg} / \mathrm{dL})$ & 90,5 & 9,3 & 92,3 & 7,4 & 91,6 & 8,2 & 0,106 \\
\hline $\mathrm{G} 2 \mathrm{~h}(\mathrm{mg} / \mathrm{dL})$ & 120,6 & 22,9 & 104,4 & 18,9 & 110,4 & 21,9 & $<0,001$ \\
\hline \multicolumn{8}{|l|}{ Grau de tolerância à glicose *** } \\
\hline Normal & 40 & 67,8 & 80 & 82,5 & 120 & 76,9 & $<0,05$ \\
\hline Glicemia de jejum alterada & 7 & 11,8 & 12 & 12,4 & 19 & 12,2 & \\
\hline Tolerância diminuída à glicose & 8 & 13,5 & 3 & 3,1 & 11 & 7,0 & \\
\hline Diabetes & 4 & 6,8 & 2 & 2,0 & 6 & 3,8 & \\
\hline Perímetro da cintura $(\mathrm{cm})$ & 85,2 & 9,2 & 87,1 & 8,1 & 86,3 & 8,6 & 0,082 \\
\hline \multicolumn{8}{|l|}{ Obesidade central \# } \\
\hline $\operatorname{Sim}$ & 43 & 67,2 & 18 & 18,2 & 61 & 37,4 & $<0,001$ \\
\hline Não & 21 & 32,8 & 81 & 81,8 & 102 & 62,6 & \\
\hline Triglicérides (mg/dL) & 157,1 & 101,3 & 186,8 & 126,5 & 177,1 & 117,8 & 0,077 \\
\hline \multicolumn{8}{|l|}{ Hipertrigliceridemia \#\# } \\
\hline $\operatorname{Sim}$ & 23 & 35,9 & 48 & 48,5 & 71 & 43,5 & 0,115 \\
\hline Não & 41 & 64,1 & 51 & 51,5 & 92 & 56,5 & \\
\hline HDL colesterol (mg/dL) & 43,5 & 11,1 & 36,6 & 9,4 & 39,3 & 10,6 & $<0,001$ \\
\hline \multicolumn{8}{|l|}{ HDL reduzido \#\#\# } \\
\hline $\operatorname{Sim}$ & 45 & 70,3 & 63 & 63,6 & 108 & 66,2 & 0,379 \\
\hline Não & 19 & 29,7 & 36 & 36,4 & 55 & 33,7 & \\
\hline \multicolumn{8}{|l|}{ Síndrome metabólica § } \\
\hline $\operatorname{Sim}$ & 26 & 40,6 & 19 & 19,4 & 45 & 27,8 & 0,003 \\
\hline Não & 38 & 59,4 & 79 & 80,6 & 117 & 72,2 & \\
\hline
\end{tabular}

GJ: glicemia de jejum; G2h: glicemia de duas horas; PAD: pressão arterial diastólica; PAS: pressão arterial sistólica.

* Qui-quadrado ou t de Student;

** Sim, se PAS e PAD forem, respectivamente, $\geq 140$ ou $90 \mathrm{mmHg}$;

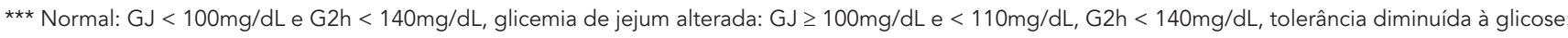

GJ < 110 e G2h entre 140 e 199mg/dL, diabetes: GJ $\geq 110 \mathrm{mg} / \mathrm{dL}$ ou G2h $\geq 200 \mathrm{mg} / \mathrm{dL}$;

\# Perímetro da cintura $>80 \mathrm{~cm}$ ou $94 \mathrm{~cm}$, para mulheres e homens, respectivamente;

\#\# Sim, se triglicérides $>150 \mathrm{mg} / \mathrm{dL}$;

\#\#\# HDL $<40 \mathrm{mg} / \mathrm{dL}$ (homens) e $<50 \mathrm{mg} / \mathrm{dL}$ (mulheres) 21;

$\S$ Sim, se o indivíduo apresenta três ou mais dos seguintes fatores de risco cardiovascular: hipertensão arterial, intolerância à glicose, obesidade central,

hipertrigliceridemia ou HDL reduzido.

\section{Discussão}

Estudos epidemiológicos têm mostrado aumento de casos de DCNT em diversas populações indígenas, evidenciando o processo de transição epidemiológica vivenciado por esses povos.
Apesar de as doenças infecciosas e parasitárias ainda serem as mais frequentes, observa-se, nos últimos anos, aumento substancial dos fatores de risco que compõem a síndrome metabólica e, consequentemente, elevação do número de doenças cardiovasculares e diabetes mellitus 10,32. 
Número (ou média), porcentagem (ou desvio-padrão - DP) e razões entre as prevalências (RP) de variáveis de interesse segundo a presença de síndrome metabólica em indígenas Khisêdjê. Parque Indígena do Xingu, Brasil, 2011.

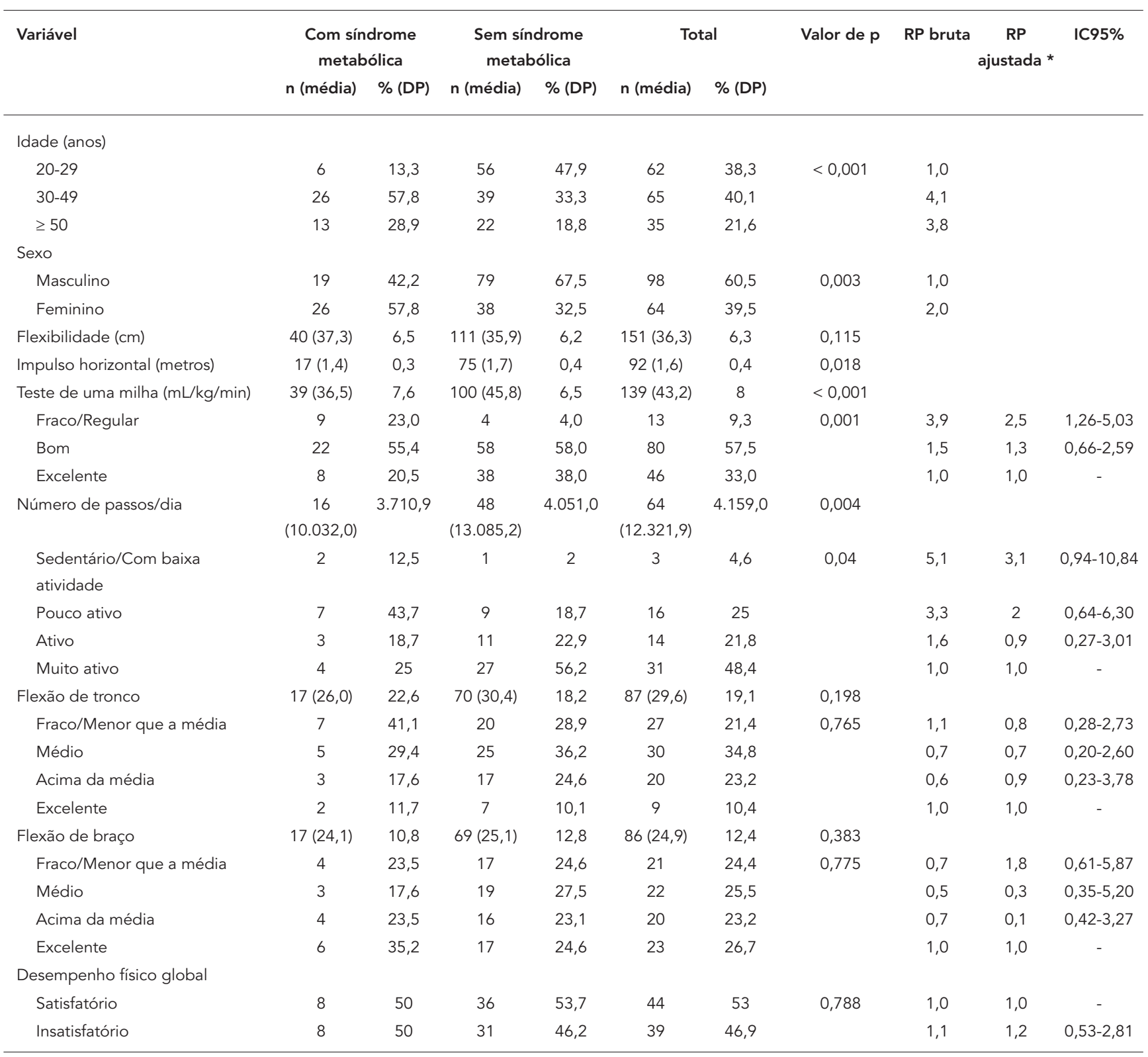

IC95\%: intervalo de 95\% de confiança; RP: razão de prevalência.

* Ajustada por sexo e idade.

Pesquisas realizadas enfatizam que o aumento da síndrome metabólica e de seus componentes está diretamente relacionado ao "processo de ocidentalização”, caracterizado por mudanças no estilo de vida, como a diminuição da atividade física diária, adoção de vícios em cigarro e bebidas alcoólicas, por exemplo, e mudanças no perfil alimentar, como o consumo excessivo de alimentos industrializados e hipercalóricos $9,33,34,35$.
No presente estudo, buscou-se avaliar a relação da atividade física com a síndrome metabólica e seus componentes. Pesquisas de natureza semelhante a esta já foram realizadas com populações urbanas; entretanto, entre indígenas, elas são raras ou inexistentes. No Brasil, este é o primeiro estudo que quantificou o nível de atividade física e condicionamento físico de indígenas e sua relação com a presença de síndrome metabólica. 
Com exceção para o teste de impulso horizontal, os resultados mostraram que a maioria dos indígenas Khisêdjê apresentou desempenho físico satisfatório nos testes físicos realizados. A presença de síndrome metabólica associou-se ao desempenho insatisfatório no teste de uma milha e ao número de passos/dia. Média significativamente maior da distância alcançada no teste de impulso horizontal (metros) foi observada entre sujeitos sem síndrome metabólica em comparação com os demais.

Em relação ao condicionamento físico, destaca-se o excelente desempenho observado nos testes de flexibilidade e de resistência cardiorrespiratória (teste de uma milha). A média geral do $\mathrm{VO}_{2 \max }$ identificada entre os Khisêdjê (43,3 $\mathrm{Ml} / \mathrm{kg} / \mathrm{min}$ ) foi semelhante à encontrada por Ciro 36 entre militares do Estado de Santa Catarina, profissão que exige rotina de treinamento físico mais intenso e, portanto, proporciona melhor condicionamento físico. Independentemente de faixa etária ou sexo, esse resultado pode ser considerado extremamente satisfatório, considerando os valores de referência para esse teste 17,24 . Contudo, a porcentagem de sujeitos com desempenho excelente foi maior no sexo masculino do que no feminino $(41,5 \%$ versus $17,4 \%)$. É provável que isso ocorra em virtude da distribuição de afazeres na aldeia, uma vez que os homens se deslocam mais que as mulheres no dia a dia, em tarefas como ir ao roçado, pescar e caçar, enquanto as mulheres exercem atividades mais próximas de casa, como artesanato, preparo dos alimentos e o cuidado com os filhos pequenos.

Em relação ao teste de flexibilidade, observou-se média geral elevada $(36,4 \mathrm{~cm}, \mathrm{DP}=6,3)$. Esse resultado se mostra satisfatório se comparado ao observado por Prati \& Prati 37 , que identificaram média de $40 \mathrm{~cm}$ em bailarinas com mais de sete anos de experiência. A flexibilidade verificada entre os Khisêdjê pode estar relacionada à posição adotada por esses indivíduos para realizar atividades cotidianas. Cozinhar, ralar e descascar a mandioca, trabalhar com artesanato, lavar roupa, deslocar-se de canoa, plantar e colher, ou apenas sentar no chão, são atividades que exigem a adoção de posturas anatômicas com elevada amplitude articular, provavelmente incômodas para outros indivíduos, mas rotineiras para os Khisêdjê.

Os resultados observados no teste de impulso horizontal não foram satisfatórios e estão aquém daqueles encontrados em outros estudos 38,39. É provável que o instrumento não seja o mais adequado para avaliar essa habilidade na população em estudo, pois é mais utilizado no rastreamento de habilidades esportivas 40 .
Os dois testes mais utilizados para avaliar a condição de força/resistência muscular são os de flexão de tronco (abdominal) e de flexão de braço 28 . A baixa aptidão nesse componente pode ser considerada um risco, pois, à medida que a idade avança, os riscos de lesões aumentam 41 . Os resultados desses testes entre os Khisêdjê se mostraram extremamente satisfatórios, sendo superiores aos observados por Prati \& Prati 37, e próximos aos encontrados por Pereira \& Teixeira 42 , em estudo com militares.

A avaliação do nível de atividade física segundo o número de passos/dia, por meio do pedômetro, demonstrou que os Khisêdjê estão em condição física extremamente satisfatória, conforme os parâmetros existentes para esta avaliação ${ }^{30}$. Tudor-Locke et al. 30 explicam que um adulto saudável costuma dar cerca de 7.000 a 13.000 passos por dia e que 30 minutos de atividade física moderada equivalem a aproximadamente 3.000 a 4.000 passos apenas. Já os Khisêdjê apresentaram média geral de 12.321,9 passos/dia, sendo considerados, em sua maioria, muito ativos ou plenamente ativos fisicamente. O resultado dessa avaliação entre os Khisêdjê demonstrou superioridade em relação aos estudos consultados, realizados com não indígenas 43,44 .

É possível que esses indivíduos tivessem, há décadas atrás, um condicionamento físico superior ao que foi revelado neste estudo. Sabe-se que a atual rotina física de trabalho e afazeres da maioria desses sujeitos se modificou, com a incorporação de alguma tecnologia (motor de barco, carro, por exemplo). Em adição, alguns já possuem trabalho remunerado na própria aldeia (agentes de saúde, professores, entre outros), o que pode refletir na sua saúde em dois aspectos: o primeiro está relacionado à diminuição da atividade física; o segundo relaciona-se ao aumento do poder de compra e consumo de alimentos industrializados desses indivíduos.

Os poucos dados disponíveis na literatura sobre atividade física entre indígenas indicam grandes desigualdades em relação a essa prática e, consequentemente, ao condicionamento físico desses indivíduos. Tais disparidades, embora possam ser influenciadas pelos diversos métodos utilizados para medir atividade física (questionários com informações sobre o tempo diário/ semanal gasto para praticar atividades; entrevistas telefônicas; avaliação do nível de atividade por meio de instrumentos como o acelerômetro), possivelmente refletem os diferentes momentos históricos e de transição vivenciados por cada etnia 33,45,46. A prevalência de indígenas ativos fisicamente varia desde $26,7 \%$ entre adultos australianos 5 até $51 \%$ em mulheres e $60 \%$ em homens na Nova Zelândia 47 . Uma pesquisa sobre fatores 
de risco para doenças crônicas identificou que $37,2 \%$ de nativos do Alasca não praticam qualquer atividade física no lazer 48.

Apesar do condicionamento físico satisfatório observado entre os Khisêdjê, encontrou-se alta prevalência de síndrome metabólica $(27,8 \%)$. Essa porcentagem foi maior que a identificada por Salvo et al. 9 quando estudaram esta mesma população anteriormente (21,9\%). Além disso, a prevalência dos componentes da síndrome metabólica entre os Khisêdjê foi superior à observada entre os Guaraní-Mbyá 7 . Oliveira et al. 49 também identificaram elevada prevalência de síndrome metabólica entre indivíduos com idade de 18 a 69 anos da etnia Jaguapirú, no Mato Grosso do Sul (43,4\% entre as mulheres e $26,1 \%$ entre os homens). Estudo realizado com nativos americanos detectou prevalências diferentes segundo o acesso a produtos industrializados pelas diferentes populações indígenas. Entre sujeitos residentes na Região Sudeste dos Estados Unidos, a prevalência foi de $43,2 \%$ e $47 \%$ entre homens e mulheres, respectivamente. Na população do Alasca, a prevalência foi de $26,5 \%$, entre os homens, e $31,2 \%$, entre as mulheres 35 . Esses achados evidenciam uma situação preocupante do quadro de síndrome metabólica, em evolução entre as populações indígenas, tornando-as mais suscetíveis às doenças cardiovasculares e diabetes mellitus.

Resultados de pesquisas epidemiológicas evidenciam a associação entre inatividade física e maior frequência da síndrome metabólica. No trabalho conduzido por Elias et al. 50, identificouse o dobro da prevalência de síndrome metabólica em indivíduos sedentários, em comparação com aqueles ativos fisicamente. Essa relação também foi confirmada no presente estudo, em que maior frequência da síndrome metabólica associou-se com desempenho insatisfatório nos testes físicos que avaliaram a força/resistência muscular localizada (membros inferiores e superiores) e nível de atividade física segundo o número de passos/dia. Esses achados são corrobo- rados por estudos que investigam os benefícios da prática regular de atividade física no controle e redução da síndrome metabólica. Katzmarzyk et al. 14 observaram redução de $30 \%$ na prevalência de síndrome metabólica em população canadense saudável após a introdução de exercícios predominantemente aeróbios.

Uma limitação do presente estudo diz respeito à pouca familiaridade dos indígenas com as técnicas de execução nos testes físicos. Tal situação talvez tenha subestimado a real condição de alguns componentes da aptidão física relacionada à saúde entre esses sujeitos. Entretanto, esse fato não elimina a importância desta investigação como um mecanismo avaliador da condição física dessas populações. De forma pioneira, desenvolveu-se uma pesquisa com esse desenho em populações indígenas, sendo possível, pela primeira vez em nosso meio, quantificar a condição física de indígenas e relacioná-la com a síndrome metabólica.

\section{Conclusão}

Neste estudo, foi possível constatar que, apesar da alta prevalência de síndrome metabólica e de seus componentes, os Khisêdjê ainda estão em condição física satisfatória, em sua maioria. $\mathrm{O}$ menor nível de atividade física e desempenho inferior nos testes físicos foram associados com maior prevalência da síndrome metabólica. A associação entre a presença de síndrome metabólica e nível insatisfatório de condicionamento físico identificada neste estudo corrobora os resultados de outros estudos realizados em populações não indígenas 14,50.

Em virtude da ausência de estudos que avaliem a condição física e sua relação com a síndrome metabólica em população indígena, não foi possível fazer comparações; todavia, os métodos, técnicas e testes utilizados nesta pesquisa podem ser reproduzidos em estudos com outras etnias. 


\section{Resumo}

Este estudo objetivou verificar a existência de associação entre o grau de atividade física e a presença de síndrome metabólica entre indígenas Khisêdjê. Foram avaliados 170 indivíduos com 20 anos ou mais. Obtiveram-se dados sociodemográficos, de testes físicos e análise de material biológico. Para análise dos dados, utilizou-se a estatística do qui-quadrado ( $p<$ 0,05 ), razões de prevalências (por ponto e por intervalo de 95\% de confiança) brutas e ajustadas e teste t de Student. Resultados satisfatórios foram observados em relação aos testes de resistência cardiorrespiratória, flexibilidade, flexão de braço e tronco, além de na avaliação do nível de atividade física segundo o número de passos/dia. A prevalência de síndrome metabólica foi de 27,8\%, sendo maior entre mulheres, entre indigenas nas faixas etárias de 39-49 anos e 50 anos ou mais e entre aqueles com desempenho inferior no teste de resistência cardiorrespiratória, impulso horizontal e número de passos/dia. Os resultados indicam a necessidade de maior vigilância no controle e prevenção dos fatores de risco que compõem a síndrome metabólica.

Síndrome X Metabólica; Atividade Motora; População Indígena

\section{Referências}

1. International Diabetes Federation. The IDF consensus worldwide definition of the metabolic syndrome. http://www.idf.org/webdata/docs/IDF_ Meta_def_final.pdf (acessado em Out/2010).

2. Nakazone MA, Nakazone MA, Pinheiro A, Braile MCVB, Pinhel MAS, Sousa GF, et al. Prevalência de síndrome metabólica em indivíduos brasileiros pelos critérios de NCEP-ATPIII e IDF. Rev Assoc Med Bras 2007; 53:407-13.

3. Picon PX, Zanatta CM, Gerchman F, Zelmanovitz T, Gross JL, Canani LH. Análise dos critérios de definição da síndrome metabólica em pacientes com diabetes melito tipo 2. Arq Bras Endocrinol Metab 2006; 50:264-70.

\section{Colaboradores}

K. M. Santos participou da coleta de dados, análise estatística e redação do manuscrito. M. L. S. Tsutsui, P. P. O. Galvão, L. Mazzucchetti e D. Rodrigues colaboraram no planejamento do estudo, coleta de dados, elaboração do banco de dados da pesquisa, interpretação dos dados e revisão do manuscrito. S. G. A. Gimeno participou do planejamento do estudo, coleta de dados, redação e revisão do manuscrito.

\section{Agradecimentos}

Aos Khisêdjê, que possibilitaram a realização deste estudo e nos acolheram com alegria e cordialidade. À FAPESP pelo financiamento da pesquisa (processo no. 2010/52263-7).
4. Snodgrass JJ, Leonard WR, Tarskaia LA, Egorova AG, Maharova NV, Pinigina IA, et al. Impaired fasting glucose and the metabolic syndrome in an in digenous Siberian population. Int J Circumpolar Health 2010; 69:87-98.

5. Li M, Campbell S, McDermott R. $\gamma$-glutamyltransferase, obesity, physical activity, and the metabolic syndrome in indigenous Australian adults. Obesity 2009; 17:809-13.

6. Rocha AKS, Bos AJG, Huttner E, Machado DC. Prevalência da síndrome metabólica em indígenas com mais de 40 anos no Rio Grande do Sul, Brasil. Rev Panam Salud Pública 2011; 29:41-5. 
7. Cardoso AM, Mattos IE, Koifman RJ. Prevalência de fatores de risco para doenças cardiovasculares na população Guaraní-Mbyá do Estado do Rio de Janeiro. Cad Saúde Pública 2001; 17:345-54.

8. Gugelmin SA, Santos RV. Uso do índice de massa corporal na avaliação do estado nutricional de adultos indígenas Xavánte, Terra Indígena Sangradouro-Volta Grande, Mato Grosso. Cad Saúde Pública 2006; 22:1865-72.

9. Salvo VLMA, Rodrigues D, Baruzzi RG, Pagliaro H, Gimeno SGA. Perfil metabólico e antropométrico dos Suyá: Parque Indígena do Xingu, Brasil Central. Rev Bras Epidemiol 2009; 12:458-68.

10. Santos RV, Coimbra Jr. CEA. Cenários e tendências da saúde e da epidemiologia dos povos indígenas no Brasil. In: Coimbra Jr. CEA, Santos RV, Escobar AL, organizadores. Epidemiologia e saúde dos povos indígenas no Brasil. Rio de Janeiro: Editora Fiocruz/ABRASCO; 2003. p. 13-47.

11. Schmidt MI, Duncan BB, Silva GA, Menezes AM, Monteiro CA, Barreto SM, et al. Doenças crônicas não transmissíveis no Brasil: carga e desafios atuais. http://download.thelancet.com/flatconten tassets/pdfs/brazil/brazilpor4.pdf (acessado em Fev/2012).

12. Castaneda C, Layne LE, Orians LM, Gordon PL, Walsmith J, Foldvari M, et al. A randomized controlled trial of resistance exercise training to improve glycemic control in older adults with type 2 diabetes. Diabetes Care 2002; 25:2335-41.

13. Ciolac EG, Guimarães GV. Exercício físico e síndrome metabólica. Rev Bras Med Esporte 2004; 10:319-24.

14. Katzmarzyk PT, Leon AS, Wilmore JH, Skinner JS, Rao DC, Rankinen T, et al. Targeting the metabolic syndrome with exercise: evidence from the HERITAGE Family Study. Med Sci Sports Exerc 2003; 35:1703-9.

15. Florindo AA. Atividade física e doenças crônicas. In: Florindo AA, Hallal PC, organizadores. Epidemiologia da atividade física. São Paulo: Editora Atheneu; 2011. p. 159-82.

16. Vuori MI. Health benefits of physical activity with special reference to interaction with diet. Public Health Nutr 2001; 4:517-28.

17. Robergs RA, Roberts SO. Princípios fundamentais de fisiologia do exercício: para aptidão, desempenho e saúde. São Paulo: Phorte; 2002.

18. Carroll S, Dudfield M. What is the relationship between exercise and metabolic abnormalities? A review of the metabolic syndrome. Sports Med 2004; 34:371-418.

19. McArdle WD, Katch FI, Katch VL. Fisiologia do exercício: energia, nutrição e desempenho humano. 6 a Ed. Rio de Janeiro: Editora Guanabara Koogan; 2008.

20. Chobanian AV, Bakris GL, Black HR, Cushman WC, Green LA, Izzo Jr. JL, et al. The Seventh Report of the Joint National Committee on Prevention, Detection, Evaluation and Treatment of High Blood Pressure: The JNC 7 Report. JAMA 2003; 289: 2560-72.
21. Alberti KGMM, Eckel RH, Grundy SM, Zimmet PZ, Cleeman JI, Donato KA, et al. Harmonizing the metabolic syndrome: a joint interim statement of the International Diabetes Federation Task Force on Epidemiology and Prevention; National Heart, Lung, and Blood Institute; American Heart Association; World Heart Federation; International Atherosclerosis Society; and International Association for the Study of Obesity. Circulation 2009; 120:1640-5.

22. American Diabetes Association. Diagnosis and classification of diabetes mellitus. Diabetes Care 2004; 27 Suppl 1:S5-10.

23. Alberti KGMM, Zimmet P. Definition, diagnosis and classification of diabetes mellitus and its complications. Part 1: diagnosis and classification of diabetes mellitus provisional report of a WHO consultation. Diabet Med 1998; 15:539-53.

24. Pitanga FJG. Testes, medidas e avaliação em educação física e esportes. $3^{a}$ Ed. São Paulo: Phorte; 2004.

25. Cardoso JR, Azevedo NCT, Cassano CS, Kawano MM, Âmbar G. Confiabilidade intra e interobservador da análise cinemática angular do quadril durante o teste sentar e alcançar para mensurar o comprimento dos isquiotibiais em universitários. Rev Bras Fisioter 2007; 11:133-8.

26. Pollock ML, Wilmore JH. Exercícios na saúde e na doença. 2a Ed. Rio de Janeiro: Editora Medsi; 1993.

27. Caldas PRL, Rocha PSO. Treinamento desportivo. Brasília: Secretaria de Educação Física e Desporto, Ministério da Educação e Cultura; 1978.

28. American College of Sports Medicine. Manual do ACSM para avaliação da aptidão física relacionada à saúde. Rio de Janeiro: Editora Guanabara Koogan; 2006.

29. Morrow JR, Jackson AW, Disch JG, Mood DP. Medida e avaliação do desempenho humano. 2a Ed. Porto Alegre: Editora Artmed; 2003.

30. Tudor-Locke C, Bell RC, Myers AM, Harris SB, Ecclestone NA, Lauzon N, et al. Controlled outcome evaluation of the first step Program: a daily physical activity intervention for individuals with type II diabetes. Int J Obes Relat Metab Disord 2004; 28:113-9.

31. Snedecor GW, Cochran WG. Statistical methods. 8th Ed. Iowa: University Press; 1989.

32. Lourenço AEP. Avaliação do estado nutricional em relação a aspectos socioeconômicos de adultos indígenas Suruí, Rondônia, Brasil [Dissertação de Mestrado]. Rio de Janeiro: Escola Nacional de Saúde Pública Sergio Arouca, Fundação Oswaldo Cruz; 2006.

33. Kriska AM, Saremi A, Hanson RL, Bennett PH, Kobes S, Williams DE, et al. Physical activity, obesity, and the incidence of type 2 diabetes in a high-risk population. Am J Epidemiol 2003; 158:669-75.

34. Gimeno SGA, Rodrigues D, Pagliaro H, Cano EM, Lima EES, Baruzzi RG. Perfil metabólico e antropométrico de índios Aruák: Mehináku, Waurá e Yawalapití, Alto Xingu, Brasil Central, 2000/2002. Cad Saúde Pública 2007; 23:1946-54. 
35. Schumacher C, Ferucci ED, Lanier AP, Slattery ML, Schraer CD, Raymer TW, et al. Metabolic syndrome: prevalence among American Indian and Alaska native people living in the southwestern United States and in Alaska. Metab Syndr Relat Disord 2008; 6:267-73.

36. Ciro RRA. Sistema de avaliação para a promoção e gestão do estilo de vida saudável e da aptidão física relacionada à saúde de policiais militares [Tese de Doutorado]. Florianópolis: Universidade Federal de Santa Catarina; 2003.

37. Prati SRA, Prati ARC. Níveis de aptidão física e análise de tendências posturais em bailarinas clássicas. Rev Bras Cineantropom Desempenho Hum 2006; 8:80-7.

38. Pereira EF, Graup S. Aptidão física relacionada à saúde e ao desempenho atlético de calouros de educação física. Efdeportes 2007; 11(104). http:// www.efdeportes.com/efd104/aptidao-fisica.htm.

39. Leite N, Aguiar Junior RP, Cieslak F, Ishiyama M, Milano GE, Stefanello JMFL. Perfil da aptidão física dos praticantes de le parkour. Rev Bras Med Esporte $2011 ; 17: 198-201$.

40. Kiss MAPDM. Avaliação em educação física. São Paulo: Editora Manole; 1987.

41. Nieman DC. Exercício e saúde. São Paulo: Editora Manole; 1999.

42. Pereira EF, Teixeira CS. Proposta de valores normativos para avaliação da aptidão física em militares da Aeronáutica. Rev Bras Educ Fís Esp 2006; 20:249-56.

43. Oliveira MM, Maia JA. Avaliação da atividade física em contextos epidemiológicos. Uma revisão da validade e fiabilidade do acelerómetro tritrac-r3d, do Pedómetro yamax digi-walker e do questionário de Baecke. Rev Port Ciênc Desporto 2001; 1: 73-88.
44. Tudor-Locke C, Burkett L, Reis JP, Ainsworth BE, Maceda CA, Wilson DR. How many days of pedometer monitoring predict weekly physical activity in adults? Prev Med 2005; 40:293-8.

45. Fretts AM, Howard BV, Kriska AM, Smith NL, Lumley T, Lee ET, et al. Physical activity and incident diabetes in American Indians: the Strong Heart Study. Am J Epidemiol 2009; 170:632-9.

46. Duncan GE, Goldberg J, Buchwald D, Wen Y, Henderson JA. Epidemiology of physical activity in American Indians in the Education and Research Towards Health cohort. Am J Prev Med 2009; 37:488-94

47. Ross J, Hamlin M. Maori physical activity: a review of an indigenous population's participation. Health Promot J Austr 2007; 18:73-6.

48. Denny CH, Holtzman D, Goins RT, Croft JB. Disparities in chronic disease risk factors and health status between American Indian/Alaska Native and White elders: findings from a telephone survey, 2001 and 2002. Am J Public Health 2005 95:825-7.

49. Oliveira GF, Oliveira TR, Rodrigues FF, Corrêa LF Arruda TB, Casulari LA. Prevalence of metabolic syndrome in the indigenous population, aged 19 to 69 years, from Jaguapiru Village, Dourados (MS), Brazil. Ethn Dis 2011; 21:301-6.

50. Elias RGM, Fernandes CAM, Fontes CER, Cuman RKN. Influência da atividade física sobre a prevalência de síndrome metabólica, em mulheres atendidas em uma unidade básica de saúde, Maringá - PR. Ciênc Cuid Saúde 2008; 7:88-93.

Recebido em 02/Mai/2012

Versão final reapresentada em 14/Ago/2012

Aprovado em 17/Ago/2012 